\title{
On the oscillation and asymptotic behavior for a kind of fractional differential equations
}

\author{
Yizhuo Wang ${ }^{1}$, Zhenlai Han ${ }^{*}$, Ping Zhao ${ }^{2}$ and Shurong Sun ${ }^{1}$
}

\author{
"Correspondence: \\ hanzhenlai@163.com \\ 'School of Mathematical Sciences, \\ University of Jinan, Jinan, Shandong \\ 250022, P.R. China \\ Full list of author information is \\ available at the end of the article
}

\begin{abstract}
In this paper, we discuss the oscillations of the fractional order differential equation $D_{a}^{\alpha} x(t)+q(t) f(x(t))=0, t \in[a,+\infty), a>0$, where $q$ is a positive real-valued function and $f$ is a continuous functional; $D_{a}^{\alpha}$ denotes the Riemann-Liouville differential operator of order $\alpha, 0<\alpha \leq 1$. We use the Riccati transformation technique to obtain some sufficient conditions which guarantee that every solution of the equation is oscillatory or the limit inferior converges to zero. Two examples are given to show the applications of our main results.
\end{abstract}

MSC: 34A08; 34K11

Keywords: oscillation; fractional differential equation; Riemann-Liouville differential operator

\section{Introduction}

The theory of fractional calculus goes back to Leibniz's note in his list to L'Hospital [1], dated 30 September 1695, in which the meaning of the derivative of order $1 / 2$ is discussed. After that in pure mathematics field the foundation of the fractional differential equations had been established. However, in recent years, many researchers found that the fractional differential equations are more accurate in describing some practical models, e.g. polymers. Today it has been used widely in physics, electrochemistry, control theory, and electromagnetic fields [2-7]. Furthermore, the fractional calculus can also provide an excellent instrument for the description of memory and hereditary properties of various materials and processes due to the existence of a 'memory' term in the model [8-13]. Since these studies there has been much research actively concerned with the fractional differential equations and many useful achievements have been obtained [14-18].

From the 1960s, a lot of books and theses about the oscillatory behavior for first, second, and higher order differential equations are presented, see [19-21]. The study of the oscillatory problem with a view on fractional differential equation is just being initiated. As a new cross-cutting area, recently some attention has been paid to oscillations of fractional differential equations [22-29].

In 2012, Chen et al. [22] studied the oscillatory behavior of the following fractional differential equation:

$$
\left[r(t)\left(D_{-}^{\alpha} y\right)^{\eta}(t)\right]^{\prime}-q(t) f\left(\int_{t}^{\infty}(v-t)^{-\alpha} y(v) d v\right)=0 \quad \text { for } t>0,
$$

O2014 Wang et al.; licensee Springer. This is an Open Access article distributed under the terms of the Creative Commons Attribution License (http://creativecommons.org/licenses/by/2.0), which permits unrestricted use, distribution, and reproduction in any medium, provided the original work is properly cited. 
where $D_{-}^{\alpha} y$ denotes the Liouville right-sided fractional derivative of order $\alpha$ with the form

$$
\left(D_{-}^{\alpha} y\right)(t):=-\frac{1}{\Gamma(1-\alpha)} \frac{d}{d t} \int_{t}^{\infty}(v-t)^{-\alpha} y(v) d v \quad \text { for } t \in \mathbb{R}_{+}:=(0, \infty) .
$$

By the Riccati transformation technique the authors obtained some sufficient conditions, which guarantee that every solution of the equation is oscillatory.

Using the same method, in 2013, Chen [23] studied oscillatory behavior of the fractional differential equation of the form

$$
\left(D_{-}^{1+\alpha} y\right)(t)-p(t)\left(D_{-}^{\alpha} y\right)(t)+q(t) f\left(\int_{t}^{\infty}(v-t)^{-\alpha} y(v) d v\right)=0 \quad \text { for } t>0,
$$

where $D_{-}^{\alpha} y$ is the Liouville right-sided fractional derivative of order $\alpha \in(0,1)$ of $y$.

Zhang [24] considered the oscillation of the nonlinear fractional differential equation with damping term,

$$
\left[a(t)\left(D_{-}^{\alpha} x(t)\right)^{\gamma}\right]^{\prime}+p(t)\left(D_{-}^{\alpha} x(t)\right)^{\gamma}-q(t) f\left(\int_{t}^{\infty}(\xi-t)^{-\alpha} x(\xi) d \xi\right)=0, \quad t \in\left[t_{0}, \infty\right),
$$

where $D_{-}^{\alpha} x(t)$ denotes the Liouville right-sided fractional derivative of order $\alpha$ of $x$. Using a generalized Riccati function and the inequality technique, he established some new oscillation criteria.

Han et al. [25] considered the oscillation for a class of fractional differential equations,

$$
\left[r(t) g\left(\left(D_{-}^{\alpha} y\right)(t)\right)\right]^{\prime}-p(t) f\left(\int_{t}^{\infty}(s-t)^{-\alpha} y(s) d s\right)=0 \quad \text { for } t>0
$$

where $0<\alpha<1$ is a real number, $D_{-}^{\alpha} y$ is the Liouville right-sided fractional derivative of order $\alpha$ of $y$. By a generalized Riccati transformation technique, oscillation criteria for the nonlinear fractional differential equation are obtained.

Qi and Huang [26] studied the oscillation behavior of the equation of the form

$$
\left(a(t)\left[r(t) D_{-}^{\alpha} x(t)\right]^{\prime}\right)^{\prime}+p(t)\left[r(t) D_{-}^{\alpha} x(t)\right]^{\prime}-q(t) \int_{t}^{\infty}(\xi-t)^{-\alpha} x(\xi) d \xi=0, \quad t \in\left[t_{0}, \infty\right),
$$

where $D_{-}^{\alpha} x(t)$ also denotes the Liouville right-sided fractional derivative and some sufficient conditions for the oscillation of the equation have been given.

The above works on the oscillation are all concerned with fractional equations with Liouville right-sided fractional derivative by the Riccati transformation technique.

We notice that very little attention is paid to oscillations of fractional differential equations with a Riemann-Liouville derivative. For work studying the oscillatory behavior of fractional differential equations with the Riemann-Liouville derivative we refer to [27, 28], and [29].

In 2012, Grace et al. [27] studied the oscillation theory for fractional differential equations by considering equations of the form

$$
D_{a}^{q} x+f_{1}(t, x)=v(t)+f_{2}(t, x), \quad \lim _{t a+} J_{a}^{1-q} x(t)=b_{1},
$$


under the conditions

$$
x f_{i}(t, x)>0 \text { for } i=1,2, x \neq 0 \text { and } t \geq a
$$

and

$$
\left|f_{1}(t, x)\right|>p_{1}(t)|x|^{\beta} \quad \text { and } \quad\left|f_{2}(t, x)\right|>p_{2}(t)|x|^{\gamma} \quad \text { for } x \neq 0 \text { and } t \geq a \text {, }
$$

where $D_{a}^{q}$ denotes the Riemann-Liouville differential operator of order $q$ with $0<q \leq 1$, and the operator $J_{a}^{p}$ is the Rieman-Liouville fractional integral operator. The authors obtained some new oscillation criteria by reducing the fractional differential equation to the equivalent Volterra fractional integral equation and by applying the inequality technique.

Marian [28] presented the oscillatory behavior of forced nonlinear fractional difference equations of the form

$$
\Delta^{\alpha} x(t)+f_{1}(t, x(t+\alpha))=v(t)+f_{2}(t, x(t+\alpha)), \quad t \in N_{0}, 0<\alpha \leq 1,\left.\Delta^{\alpha-1} x(t)\right|_{t=0}=x_{0}
$$

where $\Delta^{\alpha}$ is a Riemann-Liouville like discrete fractional difference operator of order $\alpha$, and some oscillation criteria are established by the same method in [27].

In 2013, Chen et al. [29] improved and extended some work in [27] by considering the forced oscillation of the fractional differential equation

$$
D_{a}^{q} x+f_{1}(t, x)=v(t)+f_{2}(t, x), \quad \lim _{t a+} J_{a}^{1-q} x(t)=b_{1},
$$

with the conditions

$$
D_{a}^{q-k} x(a)=b_{k} \quad(k=1,2, \ldots, m-1)
$$

and

$$
\lim _{t a+} I_{a}^{m-q} x(t)=b_{m}
$$

where $D_{a}^{q}$ denotes the Riemann-Liouville or Caputo differential operator of order $q$ with $m-1<q \leq m, m \geq 1$, and the operator $I_{a}^{m-q}$ is the Rieman-Liouville fractional integral operator. The authors obtained some new oscillation criteria by the same method as [27]. Motivated by above work, in this paper we will extend some oscillation results from integer differential equations to the fractional differential equation

$$
D_{a}^{\alpha} x(t)+q(t) f(x(t))=0, \quad t \in[a,+\infty), a>0,
$$

where $D_{a}^{\alpha}$ denotes the standard Riemann-Liouville differential operator of order $\alpha$ with $0<\alpha \leq 1, q$ is a positive real-valued function, $f$ is a continuous functional defined on $[0,+\infty) \rightarrow[0,+\infty)$ satisfying

$$
\frac{f(x)}{I^{2-\alpha} x} \geq K>0
$$

and $I^{2-\alpha}$ denotes the Riemann-Liouville integral operator. 
We will use the method of the Riccati transformation technique to study the oscillatory behavior of the fractional differential equation (1.1). To the best of our knowledge, there is not any result on the oscillation of the fractional differential equation involving the Riemann-Liouville derivative by the method of the Riccati transformation technique.

A solution of (1.1) is said to be oscillatory if it has arbitrarily large zeros on $[a,+\infty)$ and otherwise it is non-oscillatory. An equation is said to be oscillatory if all its solutions are oscillatory.

The paper is organized as follows. In the next section, we present some basic definitions of the fractional differential and integral operators, and provide some necessary lemmas. In Section 3, we mainly use the Riccati transformation technique to get some sufficient conditions which guarantee that every solution of (1.1) is oscillatory or the limit inferior converges to zero. Our results are essential new. Finally we provide some examples to show applications of our criteria.

\section{Some preliminary lemmas}

The operator $D_{a}^{\alpha}$ with $0<\alpha<1$ defined by

$$
D_{a}^{\alpha} x(t)=\frac{1}{\Gamma(1-\alpha)} \frac{d}{d t} \int_{a}^{t}(t-s)^{-\alpha} x(s) d s
$$

is called the Riemann-Liouville derivative operator. The operator $I_{a}^{\alpha}$ defined by

$$
I_{a}^{\alpha} x(t)=\frac{1}{\Gamma(\alpha)} \int_{a}^{t}(t-s)^{\alpha-1} x(s) d s
$$

is called the Riemann-Liouville integral operator. Using the integral operator $I_{a}^{\alpha}$ we can define $D_{a}^{\alpha}$ as

$$
D_{a}^{\alpha} x(t):=\frac{d}{d t} I_{a}^{1-\alpha} x(t)
$$

In general, if $n \geq 1$ is an integer and $n-1<\alpha \leq n$, then

$$
D_{a}^{\alpha} x(t):=\frac{d^{n}}{d t^{n}} I_{a}^{n-\alpha} x(t)
$$

The integral operator has the following properties, which will be used in the next lemma:

(i) $\quad I_{a}^{\alpha} I_{a}^{\beta} f(t)=I_{a}^{\alpha+\beta} f(t), \quad D_{a}^{\alpha} I_{a}^{\alpha} f(t)=f(t), \quad \alpha>0, \beta>0, f \in L(0,1)$;

(ii) $\quad \Gamma(z+1)=z \Gamma(z)$.

The Riemann-Liouville integral operator also has a general relationship between $I_{a}^{2-\alpha}$ and $I_{a}^{1-\alpha}$ like

$$
\begin{aligned}
\left(I_{a}^{2-\alpha} x(t)\right)^{\prime} & =\left(\frac{1}{\Gamma(2-\alpha)} \int_{a}^{t}(t-s)^{1-\alpha} x(s) d s\right)^{\prime} \\
& =\frac{1-\alpha}{\Gamma(2-\alpha)} \int_{a}^{t}(t-s)^{-\alpha} x(s) d s+\frac{1}{\Gamma(2-\alpha)}(t-t)^{1-\alpha} x(t)
\end{aligned}
$$




$$
\begin{aligned}
& =\frac{1}{\Gamma(1-\alpha)} \int_{a}^{t}(t-s)^{-\alpha} x(s) d s \\
& =\left(I_{a}^{1-\alpha} x\right)(t) .
\end{aligned}
$$

Lemma 2.1 [3] Let $\alpha>0$. Assume for $x: D_{0^{+}}^{\alpha} x \in L(0,1)$. Then the following equality holds:

$$
I_{0^{+}}^{\alpha} D_{0^{+}}^{\alpha} x(t)=x(t)+c_{1} t^{\alpha-1}+c_{2} t^{\alpha-2}+\cdots+c_{n} t^{\alpha-n}
$$

for some $c_{i} \in \mathbb{R}, i=1,2, \ldots, n$, where $n$ is the smallest integer greater than or equal to $\alpha$.

For more details on the Riemann-Liouville type fractional operators, see for example [2-7].

Before stating our main results, we begin with the following lemmas which are crucial in the proofs of the main results.

Lemma 2.2 Suppose that $x$ is an eventually positive solution of (1.1) and

$$
\liminf _{t \rightarrow \infty} x(t)=l \neq 0
$$

Then there is a sufficiently large $t_{2} \in[a,+\infty)$ such that

$$
I_{a}^{1-\alpha} x(t)>0 \quad \text { for } t \in\left[t_{2},+\infty\right) .
$$

Proof Let $x$ be an eventually positive solution of (1.1), which means that there exists a $t_{0} \in[a,+\infty)$ such that $x(t)>0$ for $t \in\left[t_{0},+\infty\right)$.

From the condition (2.7) we can find a $t_{1}>t_{0}$ and a constant $l^{\prime}$ with $0<l^{\prime}<l$ such that $x(t)>l^{\prime}$ for $t \in\left[t_{1},+\infty\right)$. So we can divide $I_{a}^{1-\alpha} x$ into three parts

$$
\begin{aligned}
I_{a}^{1-\alpha} x= & \frac{1}{\Gamma(1-\alpha)} \int_{a}^{t} \frac{1}{(t-s)^{\alpha}} x(s) d s \\
= & \frac{1}{\Gamma(1-\alpha)} \int_{a}^{t_{0}} \frac{1}{(t-s)^{\alpha}} x(s) d s+\frac{1}{\Gamma(1-\alpha)} \int_{t_{0}}^{t_{1}} \frac{1}{(t-s)^{\alpha}} x(s) d s \\
& +\frac{1}{\Gamma(1-\alpha)} \int_{t_{1}}^{t} \frac{1}{(t-s)^{\alpha}} x(s) d s .
\end{aligned}
$$

From Lemma 2.1 we know that if $D_{a}^{\alpha} x(t)$ exists, and this means $(t-s)^{-\alpha} x(s) \in L[a, t]$ for any $t \in[a,+\infty)$, especially $\left(t_{0}-s\right)^{-\alpha} x(s) \in L\left[a, t_{0}\right]$.

Also we get $\left|\left(t_{0}-s\right)^{-\alpha} x(s)\right| \in L\left[a, t_{0}\right]$. Therefore we can take $M=\frac{1}{\Gamma(1-\alpha)} \int_{a}^{t_{0}}\left|\frac{1}{(t-s)^{\alpha}} x(s)\right| d s$. Then we have

$$
\begin{aligned}
I_{a}^{1-\alpha} x & \geq-M+\frac{1}{\Gamma(1-\alpha)} \int_{t_{0}}^{t_{1}} \frac{1}{(t-s)^{\alpha}} x(s) d s+\frac{1}{\Gamma(1-\alpha)} \int_{t_{1}}^{t} \frac{1}{(t-s)^{\alpha}} l^{\prime} d s \\
& \geq-M+\frac{l^{\prime}\left(t-t_{1}\right)^{1-\alpha}}{\Gamma(2-\alpha)} .
\end{aligned}
$$

Obviously there exists a sufficient large $t_{2} \in\left[t_{1},+\infty\right)$ such that $\frac{l^{\prime}\left(t-t_{1}\right)^{1-\alpha}}{\Gamma(2-\alpha)}>M$. So $I_{a}^{1-\alpha} x(t)>0$ for $t \in\left[t_{2},+\infty\right)$. The proof is complete. 
Lemma 2.3 [30] If $X$ and $Y$ are nonnegative, then

$$
X^{\lambda}-\lambda X Y^{\lambda-1}+(\lambda-1) Y^{\lambda} \geq 0, \quad \text { when } \lambda>1
$$

and

$$
X^{\lambda}-\lambda X Y^{\lambda-1}-(1-\lambda) Y^{\lambda} \leq 0, \quad \text { when } 0<\lambda<1,
$$

where equality holds if and only if $X=Y$.

\section{Main results}

Theorem 3.1 If there exists a positive function $\sigma \in C^{1}(0,+\infty)$ and a sufficiently large $t_{2} \geq a$ such that

$$
\limsup _{t \rightarrow \infty} \int_{t_{2}}^{t}\left[K \sigma(s) q(s)-\frac{\left(\sigma_{+}^{\prime}(s)\right)^{2}}{4 \sigma(s)}\right] d s=\infty,
$$

where $\sigma_{+}^{\prime}(s):=\max \left\{\sigma^{\prime}(s), 0\right\}$, then every solution $x$ of $(1.1)$ is oscillatory or $\liminf _{t \rightarrow \infty} x(t)=$ 0 .

Proof Assume to the contrary that there exists a non-oscillatory solution $x$ of (1.1). Without loss of generality, we only consider the case when $x(t)$ is eventually positive, since the case when $x(t)$ is eventually negative is similar. Thus there exists $t_{0} \in(a,+\infty)$ such that $x(t)>0$ for $t \in\left[t_{0},+\infty\right)$. Next we define the 'Riccati' type function $w$ by

$$
w(t)=\sigma(t) \frac{I_{a}^{1-\alpha} x(t)}{I_{a}^{2-\alpha} x(t)} .
$$

If $\liminf _{t \rightarrow \infty} x(t) \neq 0$, from Lemma 2.2, there exists a $t_{1} \in[a,+\infty)$ such that $I_{a}^{1-\alpha} x(t)>0$ for $t>t_{1}$. Furthermore, using the same measure in Lemma 2.2, we can easily obtain the result that there exists a $t_{2} \in[a,+\infty)$ such that $I_{a}^{2-\alpha} x(t)>0$ for $t>t_{2}$. So we get $w(t)>0$ for $t \in\left[t_{2},+\infty\right)$.

Now differentiating $w(t)$ on $\left[t_{2}, \infty\right)$ we have

$$
\begin{aligned}
w^{\prime}(t) & =\sigma^{\prime}(t) \frac{I_{a}^{1-\alpha} x(t)}{I_{a}^{2-\alpha} x(t)}+\sigma(t)\left(\frac{I_{a}^{1-\alpha} x(t)}{I_{a}^{2-\alpha} x(t)}\right)^{\prime} \\
& =\frac{\sigma^{\prime}(t)}{\sigma(t)} w(t)+\sigma(t) \frac{I_{a}^{2-\alpha} x(t)\left(I_{a}^{1-\alpha} x(t)\right)^{\prime}}{\left(I_{a}^{2-\alpha} x(t)\right)^{2}}-\sigma(t) \frac{I_{a}^{1-\alpha} x(t)\left(I_{a}^{2-\alpha} x(t)\right)^{\prime}}{\left(I_{a}^{2-\alpha} x(t)\right)^{2}} \\
& =\frac{\sigma^{\prime}(t)}{\sigma(t)} w(t)+\sigma(t) \frac{D_{a}^{\alpha} x(t)}{I_{a}^{2-\alpha} x(t)}-\sigma(t) \frac{\left(I_{a}^{1-\alpha} x(t)\right)^{2}}{\left(I_{a}^{2-\alpha} x(t)\right)^{2}} \\
& =\frac{\sigma^{\prime}(t)}{\sigma(t)} w(t)-\sigma(t) \frac{q(t) f(x(t))}{I_{a}^{2-\alpha} x(t)}-\frac{w^{2}(t)}{\sigma(t)}
\end{aligned}
$$

Then using condition (1.2) we get the inequality

$$
w^{\prime}(t) \leq \frac{\sigma_{+}^{\prime}(t)}{\sigma(t)} w(t)-K \sigma(t) q(t)-\frac{w^{2}(t)}{\sigma(t)} .
$$


Now taking

$$
\lambda=2, \quad X=\frac{1}{\sigma^{\frac{1}{2}}(t)} w(t), \quad Y=\frac{\sigma_{+}^{\prime}(t)}{2 \sigma^{\frac{1}{2}}(t)},
$$

and using Lemma 2.3 and (3.3) we conclude that

$$
w^{\prime}(t) \leq-K \sigma(t) q(t)+\frac{\left(\sigma_{+}^{\prime}(t)\right)^{2}}{4 \sigma(t)}
$$

Integrating both sides from $t_{2}$ to $t$, and letting $t \rightarrow+\infty$, we have

$$
\int_{t_{2}}^{t}\left[K \sigma(s) q(s)-\frac{\left(\sigma_{+}^{\prime}(s)\right)^{2}}{4 \sigma(s)}\right] d s \leq w\left(t_{2}\right)-w(t)<w\left(t_{2}\right) .
$$

So

$$
\limsup _{t \rightarrow \infty} \int_{t_{2}}^{t}\left[K \sigma(s) q(s)-\frac{\left(\sigma_{+}^{\prime}(s)\right)^{2}}{4 \sigma(s)}\right] d s \leq w\left(t_{2}\right)<+\infty
$$

which is a contradiction to the condition (3.1) and the proof is complete.

Corollary 3.1 Assume that (1.2) hold, and there exists a sufficient large $t_{2}$ such that

$$
\limsup _{t \rightarrow \infty} \int_{t_{2}}^{t}\left[K s q(s)-\frac{1}{4 s}\right] d s=\infty
$$

Then every solution $x$ of (1.1) is either oscillatory or $\liminf _{t \rightarrow \infty} x(t)=0$.

Proof This follows from Theorem 3.1 by taking $\sigma(t)=t$.

Corollary 3.2 Assume that (1.2) hold, and there exists a sufficiently large $t_{2}$ such that

$$
\limsup _{t \rightarrow \infty} \int_{t_{2}}^{t} q(s) d s=\infty
$$

Then every solution $x$ of $(1.1)$ is either oscillatory or $\liminf _{t \rightarrow \infty} x(t)=0$.

Proof Taking $\sigma(t)=1$, then the condition (3.1) in Theorem 3.1 is reduced to (3.5). Hence the result is obtained from Theorem 3.1.

Theorem 3.2 Assume that (1.2) holds. Also, assume that there exist functions $H \in$ $C\left(D, \mathbb{R}^{+}\right), \sigma \in C^{1}(0,+\infty)$ such that

$$
H(t, t)=0 \text { and } H(t, s)>0 \text { for } t>s \geq a \text {, }
$$

where $D=\left\{(t, s) \in \mathbb{R}^{2}: t \geq s \geq a\right\}$ and $H$ has a nonpositive continuous partial derivative $H_{s}^{\prime}(t, s):=\frac{\partial H(t, s)}{\partial s}$ on $D$ with respect to the second variable. Also assume there exists a nonnegative continuous function $h$ defined on $D$ and a differentiable positive function $\sigma(t)$ satisfying for all $t \in[a,+\infty)$

$$
\frac{\sigma_{+}^{\prime}(s)}{\sigma(s)} H(t, s)+H_{s}^{\prime}(t, s)=\frac{1}{\sigma(s)} h(t, s) H^{\frac{1}{2}}(t, s),
$$


where $\sigma_{+}^{\prime}(s):=\max \left\{\sigma^{\prime}(s), 0\right\}$. If these assumptions hold and

$$
\limsup _{t \rightarrow \infty} \frac{1}{H\left(t, t_{1}\right)} \int_{t_{1}}^{t}\left[K \sigma(s) q(s) H(t, s)-\frac{h^{2}(t, s)}{4 \sigma(s)}\right] d s=\infty
$$

then every solution $x$ of $(1.1)$ is oscillatory or $\liminf _{t \rightarrow \infty} x(t)=0$.

Proof Suppose $x$ is a non-oscillatory solution of (1.1). We only consider the case that $x(t)$ is eventually positive, since the case that $x(t)$ is eventually negative is similar. Assume that $x(t)>0$ for all $t \geq t_{1}$ with large enough $t_{1}$.

If $\liminf _{t \rightarrow \infty} x(t) \neq 0$, we proceed as in the proof of Theorem 3.1 to see that (3.3) holds. Multiplying each side of (3.3) by $H(t, s)$ and integrating from $t_{1}$ to $t$, we obtain

$$
\begin{aligned}
\int_{t_{1}}^{t} K \sigma(s) q(s) H(t, s) d s \leq & -\int_{t_{1}}^{t} H(t, s) w^{\prime}(s) d s+\int_{t_{1}}^{t} H(t, s) \frac{\sigma_{+}^{\prime}(s)}{\sigma(s)} w(s) d s \\
& -\int_{t_{1}}^{t} H(t, s) \frac{1}{\sigma(s)} w^{2}(s) d s .
\end{aligned}
$$

Then using the integration by parts formula and from (3.6), (3.8) we have

$$
\begin{aligned}
\int_{t_{1}}^{t} K \sigma(s) q(s) H(t, s) d s & \\
\leq & H\left(t, t_{1}\right) w\left(t_{1}\right)+\int_{t_{1}}^{t} H_{s}^{\prime}(t, s) w(s) d s \\
& \quad+\int_{t_{1}}^{t} H(t, s) \frac{\sigma_{+}^{\prime}(s)}{\sigma(s)} w(s) d s-\int_{t_{1}}^{t} H(t, s) \frac{1}{\sigma(s)} w^{2}(s) d s \\
\leq & H\left(t, t_{1}\right) w\left(t_{1}\right)+\int_{t_{1}}^{t}\left\{\left[H_{s}^{\prime}(t, s)+H(t, s) \frac{\sigma_{+}^{\prime}(s)}{\sigma(s)}\right] w(s)-H(t, s) \frac{1}{\sigma(s)} w^{2}(s)\right\} d s \\
= & H\left(t, t_{1}\right) w\left(t_{1}\right)+\int_{t_{1}}^{t}\left\{\left[\frac{1}{\sigma(s)} h(t, s) H^{\frac{1}{2}}(t, s)\right] w(s)-H(t, s) \frac{1}{\sigma(s)} w^{2}(s)\right\} d s .
\end{aligned}
$$

Taking

$$
\lambda=2, \quad X=\left(H(t, s) \frac{1}{\sigma(s)}\right)^{\frac{1}{2}} w(s), \quad Y=\frac{h(t, s) H^{\frac{1}{2}}(t, s)}{2 H^{\frac{1}{2}}(t, s)}=\frac{h(t, s)}{2}
$$

and using Lemma 2.3 we get

$$
\int_{t_{1}}^{t} K \sigma(s) q(s) H(t, s) d s \leq H\left(t, t_{1}\right) w\left(t_{1}\right)+\int_{t_{1}}^{t} \frac{h^{2}(t, s)}{4 \sigma(s)} d s
$$

Therefore

$$
\frac{1}{H\left(t, t_{1}\right)} \int_{t_{1}}^{t}\left\{K \sigma(s) q(s) H(t, s)-\frac{h^{2}(t, s)}{4 \sigma(s)}\right\} d s \leq w\left(t_{1}\right)<+\infty
$$

which contradicts (3.7). The proof is complete. 
Theorem 3.3 Assume that (1.2) holds. Furthermore assume there is a positive function $\sigma(t)$ such that $\sigma^{\prime}(t)$ is continuous on $(0,+\infty)$ and a sufficiently large $t_{1}$ satisfies

$$
\limsup _{t \rightarrow \infty} \frac{1}{t^{m}} \int_{t_{1}}^{t}(t-s)^{m}\left[K \sigma(s) q(s)-\frac{\left(\sigma_{+}^{\prime}(s)\right)^{2}}{4 \sigma(s)}\right] d s=\infty,
$$

where $m>1$. Then every solution of (1.1) is either oscillatory or $\liminf _{t \rightarrow \infty} x(t)=0$.

Proof Suppose $x$ is a non-oscillatory solution of (1.1). We only consider the case that $x(t)$ is eventually positive, since the case that $x(t)$ is eventually negative is similar. Assume that $x(t)>0$ for all $t \geq t_{1}$ where $t_{1}$ is chosen large. If $\liminf _{t \rightarrow \infty} x(t) \neq 0$, proceeding as in Theorem 3.1 , we get

$$
w^{\prime}(t) \leq-K \sigma(t) q(t)+\frac{\left(\sigma_{+}^{\prime}(t)\right)^{2}}{4 \sigma(t)}
$$

Therefore,

$$
\int_{t_{1}}^{t}(t-s)^{m}\left[K \sigma(s) q(s)-\frac{\left(\sigma_{+}^{\prime}(s)\right)^{2}}{4 \sigma(s)}\right] d s \leq-\int_{t_{1}}^{t}(t-s)^{m} w^{\prime}(s) d s .
$$

Using the integration by parts formula leads to

$$
\begin{aligned}
\int_{t_{1}}^{t}(t-s)^{m} w^{\prime}(s) d s & =\left.(t-s)^{m} w(s)\right|_{s=t_{1}} ^{s=t}+\int_{t_{1}}^{t} m(t-s)^{m-1} w(s) d s \\
& =-\left(t-t_{1}\right)^{m} w\left(t_{1}\right)+\int_{t_{1}}^{t} m(t-s)^{m-1} w(s) d s \\
& \geq-\left(t-t_{1}\right)^{m} w\left(t_{1}\right) .
\end{aligned}
$$

Then from (3.11) we have

$$
\int_{t_{1}}^{t}(t-s)^{m}\left[K \sigma(s) q(s)-\frac{\left(\sigma_{+}^{\prime}(s)\right)^{2}}{4 \sigma(s)}\right] d s \leq\left(t-t_{1}\right)^{m} w\left(t_{1}\right),
$$

and so

$$
\frac{1}{t^{m}} \int_{t_{1}}^{t}(t-s)^{m}\left[K \sigma(s) q(s)-\frac{\left(\sigma_{+}^{\prime}(s)\right)^{2}}{4 \sigma(s)}\right] d s \leq\left(\frac{t-t_{1}}{t}\right)^{m} w\left(t_{1}\right) .
$$

Hence,

$$
\limsup _{t \rightarrow \infty} \frac{1}{t^{m}} \int_{t_{1}}^{t}(t-s)^{m}\left[K \sigma(s) q(s)-\frac{\left(\sigma_{+}^{\prime}(s)\right)^{2}}{4 \sigma(s)}\right] d s \leq w\left(t_{1}\right),
$$

which is a contradiction of (3.10). So the proof is complete.

\section{Examples}

In this section, we will show applications of our main results. 
Example 4.1 Consider the fractional differential equation

$$
D_{a}^{\alpha} x(t)+\frac{1}{\sqrt{t}} \int_{a}^{t} \frac{1}{\Gamma(2-\alpha)}(t-s)^{1-\alpha} x(s) d s=0, \quad t>a>0,
$$

where $\alpha \in(0,1), D_{a}^{\alpha}$ is the Riemann-Liouville differential operator. In (4.1), $q(t)=\frac{1}{\sqrt{t}}$, $f(x(t))=\int_{a}^{t} \frac{1}{\Gamma(2-\alpha)}(t-s)^{1-\alpha} x(s) d s$. Set $K=1$. Then $\frac{f(x)}{I^{2-\alpha} x} \geq K>0$. Taking $\sigma(s)=s$, we obtain

$$
\begin{aligned}
& \limsup _{t \rightarrow \infty} \int_{t_{1}}^{t}\left[K \sigma(s) q(s)-\frac{\left(\sigma_{+}^{\prime}(s)\right)^{2}}{4 \sigma(s)}\right] d s \\
& \quad=\limsup _{t \rightarrow \infty} \int_{t_{1}}^{t}\left[\sqrt{s}-\frac{1}{4 s}\right] d s \\
& \quad=\infty,
\end{aligned}
$$

which implies that all conditions in Theorem 3.1 hold. So by Theorem 3.1 every solution of (4.1) is oscillatory or $\liminf _{t \rightarrow \infty} x(t)=0$.

Example 4.2 Consider the fractional differential equation

$$
D_{a}^{\alpha} x(t)+e^{t} \int_{a}^{t} \frac{1}{\Gamma(2-\alpha)}(t-s)^{1-\alpha} x(s) d s=0, \quad t>a,
$$

where $\alpha \in(0,1), D_{a}^{\alpha}$ is the Riemann-Liouville differential operator. In (4.2), $q(t)=e^{t}$, $f(x(t))=\int_{a}^{t} \frac{1}{\Gamma(2-\alpha)}(t-s)^{1-\alpha} x(s) d s$. Set $K=1$. Then $\frac{f(x)}{I^{2-\alpha} x} \geq K>0$. Taking $\sigma(s)=1$, and $m=2$ we obtain

$$
\begin{aligned}
& \limsup _{t \rightarrow \infty} \frac{1}{t^{m}} \int_{t_{1}}^{t}(t-s)^{m}\left[K \sigma(s) q(s)-\frac{\left(\sigma_{+}^{\prime}(s)\right)^{2}}{4 \sigma(s)}\right] d s \\
& \quad=\limsup _{t \rightarrow \infty} \frac{1}{t^{2}} \int_{t_{1}}^{t}(t-s)^{2} e^{s} d s \\
& \quad=\limsup _{t \rightarrow \infty} \frac{1}{t^{2}}\left[\left.(t-s)^{2} e^{s}\right|_{t_{1}} ^{t}+\int_{t_{1}}^{t} 2(t-s) e^{s} d s\right] \\
& \quad=\limsup _{t \rightarrow \infty} \frac{1}{t^{2}}\left[-\left(t-t_{1}\right)^{2} e^{t_{1}}+\left.2(t-s) e^{s}\right|_{t_{1}} ^{t}+\left.2 e^{s}\right|_{t_{1}} ^{t}\right] \\
& \quad=\limsup _{t \rightarrow \infty} \frac{1}{t^{2}}\left[-\left(t-t_{1}\right)^{2} e^{t_{1}}-2\left(t-t_{1}\right) e^{t_{1}}+2 e^{t}-2 e^{t_{1}}\right] \\
& =\infty
\end{aligned}
$$

which yields the result that all conditions on Theorem 3.3 hold. Therefore, by Theorem 3.3 every solution of (4.2) is oscillatory or $\liminf _{t \rightarrow \infty} x(t)=0$. 


\author{
Author details \\ 'School of Mathematical Sciences, University of Jinan, Jinan, Shandong 250022, P.R. China. ${ }^{2}$ School of Electrical \\ Engineering, University of Jinan, Jinan, Shandong 250022, P.R. China.
}

\title{
Acknowledgements
}

This research is supported by the Natural Science Foundation of China (61374074), Natural Science Outstanding Youth Foundation of Shandong Province (JQ201119) and supported by Shandong Provincial Natural Science Foundation (ZR2012AM009, ZR2013AL003), also supported by Natural Science Foundation of Educational Department of Shandong Province (J11LA01)

\section{Received: 29 September 2013 Accepted: 13 January 2014 Published: 31 Jan 2014}

\section{References}

1. Leibniz, GW: Mathematische Schriften. Georg Olms Verlagsbuchhandlung, Hildesheim (1962)

2. Diethelm, K: The Analysis of Fractional Differential Equations. Springer, Berlin (2010)

3. Kilbas, AA, Srivastava, HM, Trujillo, JJ: Theory and Applications of Fractional Differential Equations. Elsevier, Amsterdam (2006)

4. Podlubny, I: Fractional Differential Equations. Academic Press, San Diego (1999)

5. Das, S: Functional Fractional Calculus for System Identification and Controls. Springer, New York (2008)

6. Oldham, KB, Spanier, J: The Fractional Calculus. Academic Press, San Diego (1974)

7. Miller, KS, Ross, B: An Introduction to the Fractional Calculus and Fractional Differential Equations. Wiley, New York (1993)

8. Metzler, R, Schick, S, Kilian, HG, Nonnenmacher, TF: Relaxation in filled polymers: a fractional calculus approach J. Chem. Phys. 103, 7180-7186 (1995)

9. Glöckle, WG, Nonnenmacher, TF: A fractional calculus approach to self similar protein dynamics. Biophys. J. 68, 46-53 (1995)

10. Diethelm, K, Freed, AD: Fractional calculus in biomechanics: a 3D viscoelastic model using regularized fractional-derivative kernels with application to the human calcaneal fat pad. Biomech. Model. Mechanobiol. 5 203-215 (2006)

11. Hilfer, R: Applications of Fractional Calculus in Physics. World Scientific, River Edge (2000)

12. Magin, RL: Fractional calculus in bioengineering. Crit. Rev. Biomed. Eng. 32, 1-377 (2004)

13. Baillie, RT: Long memory processes and fractional integration in econometrics. J. Econ. 73, 5-59 (1996)

14. Diethelm, K, Ford, NJ: Analysis of fractional differential equations. J. Math. Anal. Appl. 265, 229-248 (2002)

15. Cabada, A, Wang, GT: Positive solutions of nonlinear fractional differential equations with integral boundary value conditions. J. Math. Anal. Appl. 389, 403-411 (2012)

16. Sun, S, Zhao, Y, Han, Z, Li, Y: The existence of solutions for boundary value problem of fractional hybrid differential equations. Commun. Nonlinear Sci. Numer. Simul. 17, 4961-4967 (2012)

17. Galeone, L, Garrappa, R: Explicit methods for fractional differential equations and their stability properties. J. Comput. Appl. Math. 288, 548-560 (2009)

18. Zhang, $X, L i u, L, W u, Y$ : Multiple positive solutions of a singular fractional differential equation with negatively perturbed term. Math. Comput. Model. 55, 1263-1274 (2012)

19. Han, Z, Sun, S, Shi, B: Oscillation criteria for a class of second order Emden-Fowler delay dynamic equations on time scales. J. Math. Anal. Appl. 334, 847-858 (2007)

20. Bohner, M, Erbe, L, Peterson, A: Oscillation for nonlinear second order dynamic equations on a time scale. J. Math. Anal. Appl. 301, 491-507 (2005)

21. Erbe, L, Peterson, A: Boundedness and oscillation for nonlinear dynamic equations on a time scale. Proc. Am. Math. Soc. 132, 735-744 (2004)

22. Chen, D: Oscillation criteria of fractional differential equations. Adv. Differ. Equ. 2012, 33 (2012)

23. Chen, D: Oscillatory behavior of a class of fractional differential equations with damping. Univ. Politeh. Bucharest Sci. Bull. 75, 107-118 (2013)

24. Zheng, B: Oscillation for a class of nonlinear fractional differential equations with damping term. J. Adv. Math. Stud. 6 107-115 (2013)

25. Han, Z, Zhao, Y, Sun, Y, Zhang, C: Oscillation for a class of fractional differential equation. Discrete Dyn. Nat. Soc. 2013, Article ID 390282 (2013)

26. Qi, C, Huang, S: Interval oscillation criteria for a class of fractional differential equations with damping term. Math Probl. Eng. 2013, Article ID 301085 (2013). doi:10.1155/2013/301085

27. Grace, SR, Agarwal, RP, Wong, JY, Zafer, A: On the oscillation of fractional differential equations. Fract. Calc. Appl. Anal. 15, 222-231 (2012)

28. Marian, SL: Oscillation of fractional nonlinear difference equations. Math. Æterna 2, 805-813 (2012)

29. Chen, D, Qu, P, Lan, Y: Forced oscillation of certain fractional differential equations. Adv. Differ. Equ. 2013,125 (2013)

30. Hardy, GH, Littlewood, JE, Pólya, G: Inequalities. Cambridge University Press, Cambridge (1959)

10.1186/1687-1847-2014-50

Cite this article as: Wang et al.: On the oscillation and asymptotic behavior for a kind of fractional differential equations. Advances in Difference Equations 2014, 2014:50 